OPEN ACCESS

Edited by:

David Hassel,

Heidelberg University,

Germany

Reviewed by:

Thorsten Kessler,

Deutsches Herzzentrum München,

Germany

Hauke Busch,

University of Lübeck, Germany

${ }^{*}$ Correspondence:

Tanja Zeller

t.zeller@uke.de

tThese authors have contributed equally to this work.

Specialty section: This article was submitted to Cardiovascular Genetics and

Systems Medicine,

a section of the journal

Frontiers in Cardiovascular Medicine

Received: 10 July 2017

Accepted: 30 August 2017

Published: 20 September 2017

Citation:

Zeller T, Seiffert M, Müller $C$, Scholz M, Schäffer A, Ojeda F, Drexel $H$, Mündlein A, Kleber ME, März W, Sinning C, Brunner FJ, Waldeyer C, Keller T, Saely CH, Sydow K, Thiery J, Teupser D, Blankenberg $S$ and Schnabel $R$ (2017) Genome-Wide Association Analysis for Severity of Coronary Artery Disease Using the Gensini Scoring System.

Front. Cardiovasc. Med. 4:57. doi: 10.3389/fcvm.2017.00057

\section{Genome-Wide Association Analysis for Severity of Coronary Artery Disease Using the Gensini Scoring System}

\author{
Tanja Zeller ${ }^{1,2 * t}$, Moritz Seiffert',2†, Christian Müller ${ }^{1,2}$, Markus Scholz $^{3,4}$, Anna Schäffer ${ }^{1}$, \\ Francisco Ojeda', Heinz Drexel ${ }^{5,6,7}$, Axel Mündlein $^{5}$, Marcus E. Kleber ${ }^{3}$, Winfried März ${ }^{8,9,10}$, \\ Christoph Sinning ${ }^{1}$, Fabian J. Brunner ${ }^{1}$, Christoph Waldeyer ${ }^{1}$, Till Keller ${ }^{11,12}$, \\ Christoph H. Saely ${ }^{5,6,13}$, Karsten Sydow', Joachim Thiery ${ }^{4,14}$, Daniel Teupser ${ }^{4,15}$, \\ Stefan Blankenberg ${ }^{1,2}$ and Renate Schnabel ${ }^{1,2}$ \\ ${ }^{1}$ Department of General and Interventional Cardiology, University Heart Center Hamburg, Hamburg, Germany, \\ ${ }^{2}$ DZHK (German Centre for Cardiovascular Research), Partner Site Hamburg/Kiel/Lübeck, Hamburg, Germany, \\ ${ }^{3}$ Institute for Medical Informatics, Statistics and Epidemiology, University of Leipzig, Leipzig, Germany, ${ }^{4}$ LIFE - Leipzig \\ Research Center for Civilization Diseases, University of Leipzig, Leipzig, Germany, ${ }^{5}$ Vorarlberg Institute for Vascular \\ Investigation and Treatment (VIVIT), Feldkirch, Austria, ${ }^{6}$ Private University of the Principality of Liechtenstein, Triesen, \\ Liechtenstein, ${ }^{7}$ Drexel University College of Medicine, Philadelphia, PA, United States, ${ }^{8}$ Mannheim Medical Faculty, 5th \\ Department of Medicine Medicine (Nephrology, Hypertensiology, Endocrinology, Diabetology, Rheumatology), Heidelberg \\ University, Mannheim, Germany, ${ }^{9}$ Synlab Academy, Synlab Holding Deutschland GmbH, Mannheim, Germany, \\ ${ }^{10} \mathrm{Clinical}$ Institute of Medical and Chemical Laboratory Diagnostics, Medical University of Graz, Graz, Austria, ${ }^{11}$ Kerckhoff \\ Heart and Thorax Center, Department of Cardiology, Bad Nauheim, Germany, ${ }^{12}$ German Centre for Cardiovascular \\ Research (DZHK), Partner Site RheinMain, Bad Nauheim, Germany, ${ }^{13}$ Department of Medicine and Cardiology, Academic \\ Teaching Hospital Feldkirch, Feldkirch, Austria, ${ }^{14}$ Institute of Laboratory Medicine, Clinical Chemistry and Molecular \\ Diagnostics, University of Leipzig, Leipzig, Germany, ${ }^{15}$ Institute of Laboratory Medicine, University Hospital Munich (LMU) \\ and Ludwig-Maximilian-University Munich, Munich, Germany
}

Coronary artery disease (CAD) has a complex etiology involving numerous environmental and genetic factors of disease risk. To date, the genetic 9p21 locus represents the most robust genetic finding for prevalent and incident CAD. However, limited information is available on the genetic background of the severity and distribution of CAD. CAD manifests itself as stable CAD or acute coronary syndrome. The Gensini score quantifies the extent CAD but requires coronary angiography. Here, we aimed to identify novel genetic variants associated with Gensini score severity and distribution of CAD. A two-stage approach including a discovery and a replication stage was used to assess genetic variants. In the discovery phase, a meta-analysis of genome-wide association data of 4,930 CAD-subjects assessed by the Gensini score was performed. Selected single nucleotide polymorphisms (SNPs) were replicated in 2,283 CAD-subjects by de novo genotyping. We identified genetic loci located on chromosome 2 and 9 to be associated with Gensini score severity and distribution of CAD in the discovery stage. Although the loci on chromosome 2 could not be replicated in the second stage, the known CADlocus on chromosome 9p21, represented by rs133349, was identified and, thus, was confirmed as risk locus for CAD severity.

Keywords: genetics, Gensini score, polymorphism, genome-wide association, severity of coronary artery disease 


\section{INTRODUCTION}

Coronary artery disease (CAD) is the most common cause of death in industrialized countries, and its prevalence is rapidly increasing in developing countries. CAD has a complex and heterogeneous etiology involving numerous environmental and genetic factors of disease risk. According to epidemiological and family-based studies, the genetic heritability of CAD is suggested to be $\sim 40-60 \%$ within a population (1). Common genetic susceptibility loci have been identified to be independently associated with CAD risk through genome-wide association studies (GWAS) $(2,3)$, including the $9 \mathrm{p} 21$ locus as being the most robust genetic finding for CAD to date. However, from a clinical perspective the observed effect sizes of these loci are small to moderate (3) which may, in part, be due to the heterogeneity of the different CAD phenotypes that were examined ranging from population at risk of developing CAD over anamnestic CAD to angiographically confirmed $\mathrm{CAD}$ and from stable disease to acute coronary syndrome.

In primary prevention, population risk of CAD is established mainly by the Framingham Risk Score (4). However, in recent years, this attributable risk was refined by additional markers indicating low, intermediate, or high risk populations (5). Coronary angiography remains the gold standard to assess CAD (6). The Gensini score (7) was established to quantify the severity and extent of CAD, including stenosis severity and anatomical location according to its functional relevance in the coronary circulation. An important advantage of using the Gensini score over others (e.g., SYNTAX score) is that it includes early alterations of atherosclerotic disease and mild stenoses.

In this study, we aimed to identify additional genetic loci more specific to the severity and distribution of CAD. We carried out a genome-wide approach of genetic variations with the Gensini score in European patients who underwent coronary angiography.

\section{MATERIALS AND METHODS}

\section{GWA Meta-analysis-Discovery}

We conducted a meta-analysis of GWAS data in 4,931 participants of European ancestry from three large cohorts. The study was performed in the AtheroGene study (8), the LIFE Heart Study (9), and the LURIC study (10). Each study was carried out in accordance with the recommendations of the local ethics committee, and all participants gave written informed consent in accordance with the Declaration of Helsinki. The protocols were approved by the respective local ethics and data safety committee. The cohorts are described in detail in the Supplementary Material.

\section{Assessment of the Gensini Score}

Coronary artery disease severity was assessed according to the method described by Gensini (7). Briefly, each coronary artery lesion is scored for the diameter stenosis $(25 \%=1,50 \%=2$, $75 \%=4,90 \%=8,99 \%=16$, complete occlusion) This score is multiplied with a predefined factor according to the functional relevance of the diseased vascular segment. This calculation yields a sum score describing CAD severity in each patient.

\section{Genotyping and Imputation}

Genotyping was conducted using the Affymetrix Whole-Genome Human SNP Array 6.0 in AtheroGene and LURIC and using the Affymetrix Axiom CADLIFE Array in LIFE Heart. Processing of DNA samples was performed using the Affymetrix GenomeWide Human SNP Nsp/Sty Assay 5.0/6.0, and the Affymetrix/ Axiom Reagent kit, respectively, and hybridization was done in accordance with the manufacturers' standard recommendations. Details on quality control used for genotyping are described in the Supplementary Material.

Each study imputed genotype data to 2.5 million nonmonomorphic, autosomal SNPs using HapMap haplotypes (CEU population, release 24, build 36 ) as reference. Imputation was performed with the software IMPUTE v2.1.0. as reference. Analyses were restricted to individuals of European descent only.

\section{Statistical Analyses}

Genome-wide association study was conducted in each discovery cohort independently. All individuals with coronary artery bypasses or with a Gensini score $=0$ were excluded from analysis to ensure that the observed effect was driven by the continuous severity of CAD rather than binary presence or absence of the disease. Data were analyzed by applying linear regression with an additive genetic model ( 1 degree of freedom trend test) to evaluate the association between log-transformed Gensini scores and genotypes ( 0,1 , and 2 variant alleles). Analyses were performed with adjustment for age and gender in all studies. To account for population stratification, we additionally adjusted for the first three components from principal component analysis (PCA). A fixed-effects meta-analysis was conducted by combining individual estimates of genotype effects from AtheroGene, LIFE Heart, and LURIC after excluding genotyped and imputed SNPs not meeting the quality control filters (Supplementary Material).

An a priori threshold for genome-wide significance was $5 \times 10^{-8}$, and a $p$-value $>5 \times 10^{-8}$ but $<5 \times 10^{-6}$ was considered moderate evidence for association.

\section{Replication}

Replication cohorts included 2,283 participants from the VIVIT, stenoCardia, and INTERCATH studies. Details about these cohorts are provided in the Supplementary Material.

For replication phase, we selected SNPs with a $p$-value $<5 \times 10^{-6}$ from the discovery meta-analysis (rs1485086, rs 2376012, rs16835318, and rs17752803) on chromosome 2 and SNP rs1333049 on chromosome 9.

De novo genotyping for replication was performed for the five selected SNPs in VIVIT, stenoCardia and INTERCATH by $5^{\prime}$ Nuclease assays using $\mathrm{ABI}$ genotyping assays (Applied Biosystems, Darmstadt, Germany). Genotyping was performed in 96-well plates. The Genotyping Master Mix (Applied Biosystems, Darmstadt, Germany) was used in a $7-\mu$ l total reaction volume, including $20 \mathrm{ng}$ DNA per reaction. Genotypes were automatically attributed by measuring the allele-specific fluorescence on the ABI 7900HT real-time PCR System (Applied Biosystems, Darmstadt, Germany), using the SDS 2.4 software for allele discrimination (auto caller confidence interval $>95 \%$ ). 
Association was tested by a linear regression model assuming additive allele effects in the replication studies. For single SNP replication analysis, we assumed concordant direction of effect and a $p$-value $<0.05$. Combined meta-analysis was repeated for the replication cohorts alone.

\section{RESULTS}

The characteristics of the discovery and replication cohorts are presented in Table 1. Mean age of the study participants in the discovery cohorts was 63 years $(S D \pm 9.8)$ in AtheroGene, 63.4 years $(S D \pm 11.3)$ in LIFE Heart, and 62.3 years $(S D \pm 10.8)$ in LURIC. 22, 26.6, and 31.8\% of the participants, respectively, were women. The median Gensini score was 38 (18-65.5) in AtheroGene, 24 (6-49) in LIFE Heart, and 27 (5-53.5) in LURIC.

In the replication cohorts, mean age was 64.2 years $(S D \pm 10.5)$ in Vivit, 64 years $(S D \pm 11)$ in stenoCardia, and 69.7 years $(\mathrm{SD} \pm 10.6)$ in INTERCATH. $27.8,29.4$, and $27.8 \%$ of the participants, respectively, were women. The median Gensini score was 15 (1-42) in Vivit, 23 (9-48) in stenoCardia, and 16 (6.5-38) in INTERCATH.

Figure 1 provides a Manhattan plot of the meta-analysis $p$-values by chromosomal positions. Results of the meta-analysis

TABLE 1 | Characteristics of study participants of the three discovery cohorts in the meta-analysis of genome-wide association studies of severity of coronary artery disease using the Gensini score.

\begin{tabular}{|c|c|c|c|c|c|c|}
\hline & \multicolumn{3}{|c|}{ Discovery } & \multicolumn{3}{|c|}{ Replication } \\
\hline & $\begin{array}{c}\text { AtheroGene } \\
n=1,168\end{array}$ & $\begin{array}{l}\text { LIFE Heart } \\
n=1,603\end{array}$ & $\begin{array}{c}\text { LURIC } \\
n=1,899\end{array}$ & $\begin{array}{c}\text { Vivit } \\
n=1,235\end{array}$ & $\begin{array}{c}\text { stenoCardia } \\
n=490\end{array}$ & $\begin{array}{c}\text { INTERCATH } \\
n=546\end{array}$ \\
\hline Age, years, mean $\pm S D$ & $63 \pm 9.8$ & $63.4 \pm 11.3$ & $62.3 \pm 10.8$ & $64.2 \pm 10.5$ & $64 \pm 11$ & $69.7 \pm 10.6$ \\
\hline Women, \% & $257(22)$ & 427 (26.6) & $603(31.8)$ & $343(27.8)$ & $144(29.4)$ & $151(27.8)$ \\
\hline Gensini score, median (25th-75th percentile) & $38(18-65.5)$ & $24(6-48)$ & $27(5-53.5)$ & $15(1-42)$ & $23(9-48)$ & $16(6.5-38)$ \\
\hline Hypertension, \% & $838(71.7)$ & $1067(66.6)$ & $1364(71.8)$ & $1012(82.0)$ & $383(78.2)$ & $429(81.2)$ \\
\hline Diabetes mellitus, $N(\%)$ & $248(21.2)$ & 533 (33.3) & 746 (39.3) & $366(29.7)$ & $83(16.9)$ & $151(28.3)$ \\
\hline Current cigarette smoker, $N(\%)$ & $366(31.3)$ & $421(26.3)$ & $455(24.0)$ & $229(18.6)$ & $147(30.1)$ & $84(16.5)$ \\
\hline Former cigarette smoker, $N(\%)$ & $363(31.1)$ & $624(38.9)$ & $723(38.1)$ & $535(43.4)$ & $140(29.1)$ & 249 (48.9) \\
\hline Total cholesterol, mg/dL, mean \pm SD & $215.0 \pm 48.5$ & $207.0 \pm 47.5$ & $193.4 \pm 38.9$ & $204.7 \pm 46.9$ & $209.2 \pm 47.0$ & $167.7 \pm 47.7$ \\
\hline HDL cholesterol, mg/dL, mean \pm SD & $48.8 \pm 14.4$ & $47.5 \pm 13.6$ & $39.0 \pm 10.9$ & $52.6 \pm 15.5$ & $48.5 \pm 13.6$ & $47.8 \pm 21.2$ \\
\hline LDL cholesterol, mg/dL, mean \pm SD & $138.8 \pm 43$ & $129 \pm 42$ & $116.6 \pm 34.4$ & $128.03 \pm 39.12$ & $130.2 \pm 42.2$ & $93.6 \pm 38.6$ \\
\hline Triglycerides, mg/dL, mean \pm SD & $165.37 \pm 114.6$ & $173 \pm 108$ & $170.9 \pm 121.6$ & $151.6 \pm 99.8$ & $162.2 \pm 123.9$ & $153.8 \pm 195.7$ \\
\hline Body mass index, $\mathrm{kg} / \mathrm{m}^{2}$, mean $\pm \mathrm{SD}$ & $27.5 \pm 3.7$ & $29.5 \pm 4.8$ & $27.5 \pm 4.1$ & $27.4 \pm 4.2$ & $27.9 \pm 4.2$ & $27.2 \pm 5.2$ \\
\hline History of Ml, N (\%) & $280(24)$ & $400(25)$ & $710(37.4)$ & 363 (29.3) & $95(19.8)$ & $123(23.5)$ \\
\hline
\end{tabular}

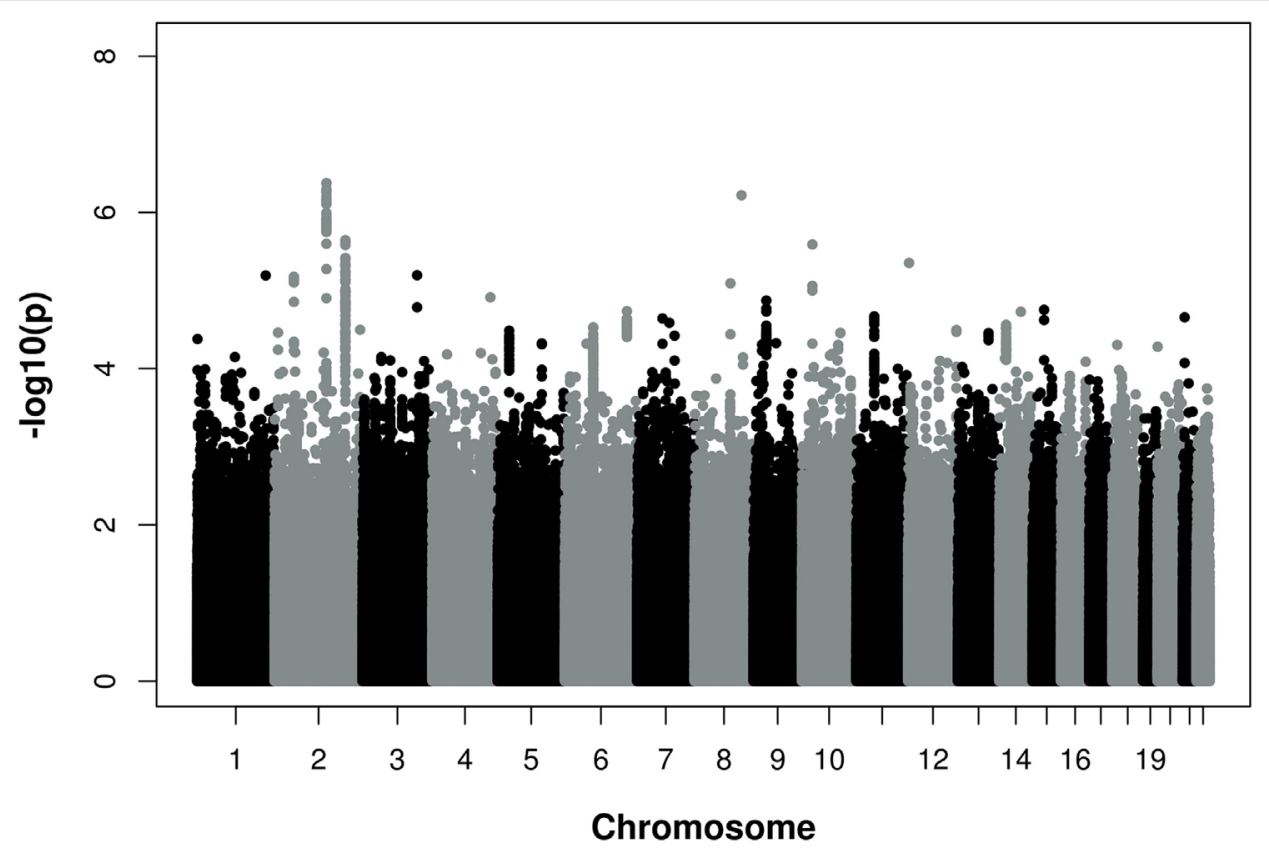

FIGURE 1 | Manhattan Plot of -log10 (p) for association of single nucleotide polymorphisms (SNPs) and chromosomal position for all autosomal SNPs analyzed in the meta-analysis of three independent discovery cohorts. Associations with a $p$-value $<5 \times 10^{-8}$ were considered genome-wide significant. The analysis was adjusted for age and gender. 
are shown in Table S1 in Supplementary Material. None of the SNPs reached genome-wide significance according to the conservative Bonferroni threshold of $P<5 \times 10^{-8}$. However, two loci in an intergenic region on chromosome 2 (2q22.1 and $2 \mathrm{q} 32.3)$ reached $p$-values of $<5 \times 10^{-6}$. The $2 \mathrm{q} 22.1$ region was represented by rs1485086-C $\left[p=7.8 \times 10^{-7}\right.$, beta $=0.196(\mathrm{SD}$ $0.04)], \mathrm{rs} 2376012-\mathrm{A}\left[p=7.7 \times 10^{-7}\right.$, beta $\left.=0.128(\mathrm{SD} 0.026)\right]$, rs17752803-T $\left[p=4.2 \times 10^{-7}\right.$, beta $=-0.214$ (SD 0.042)], and $2 \mathrm{q} 32.2$ by rs16835318-A $\left[p=3.8 \times 10^{-6}\right.$, beta $\left.=0.191(\mathrm{SD} 0.041)\right]$. In addition, on chromosome 9p21, the most significant and known CAD locus so far, several SNPs showed an association with CAD severity. These SNPs included rs4977575-G $\left[1.3 \times 10^{-5}\right.$, beta $=0.103($ SD 0.024) $]$, rs1333047-T $\left[1.7 \times 10^{-5}\right.$, beta $=0.102$ $(\mathrm{SD} 0.024)]$, and $\mathrm{rs} 1333049-\mathrm{C}\left[1.9 \times 10^{-5}\right.$, beta $=0.101(\mathrm{SD}$ $0.024)$ ].

To validate the regions on chromosome 2, the four SNPs (rs1485086, rs2376012, rs16835318, and rs17752803) were selected for de novo genotyping in our replication cohorts. In addition, we assessed SNP rs1333049 on chromosome 9 (as representative SNP of this known CAD locus) in our replication cohorts.

None of the four SNPs on chromosome 2 reached the predefined statistical significance for replication and, thus, could not be considered as replicated neither as single SNPs nor in the combined meta-analysis. However, the locus on chromosome 9 p21 reached the statistical significance threshold for replication and was confirmed as locus for the severity of stenosis in CAD [rs1333049-C, $p=1.27 \times 10^{-2}$, beta $=0.062($ SD 0.025)] The results of the replication and combined analysis are listed in Table 2 and Figure 2.

\section{DISCUSSION}

Our genome-wide association (GWA) study of angiographically defined CAD revealed that the well-established 9p21 locus is not only associated with disease risk but also severity and extent of CAD. However, this refined CAD phenotype did not lead to the identification of novel loci beyond this known locus in our medium-sized study samples.
Coronary angiography remains the gold standard for the assessment of CAD at current stage. A continuous acquisition of $\mathrm{CAD}$ severity may be superior to a binary assessment of CAD presence due to its progressive nature over time. Scoring systems such as the Gensini score (7) reflect the severity and extent of $\mathrm{CAD}$ and precisely define the disease phenotype. With the progression of CAD, an individual's Gensini score may increase over time. Accordingly, analyses were adjusted for age of the patients. Prognostic implications of the Gensini score on overall survival have been demonstrated (11). CAD is a heterogeneous phenotype that is not fully mirrored by most of the recent GWAS (12). For example, high heritability estimates have been reported for leftmain disease and calcified coronary lesions (13). The Gensini score provides a detailed representation across the spectrum of CAD as it already reflects minor angiographically detectable vascular alterations and, thus, should more accurately subclassify individuals with a diagnosis of CAD.

Recent studies have demonstrated an association between 9p21 and CAD burden with number of diseased vessels or semiquantitative methods such as the Gensini score, suggesting that 9p21 promotes progressive atherosclerosis (14-18). By contrast, other studies have not confirmed this association, and this lack of consistency has led to difficulties in reconciling association with presence but not extent of CAD $(19,20)$.

A recent large meta-analysis, which included a breadth of published and unpublished reports on 9p21 and angiographic CAD, convincingly demonstrated that $9 \mathrm{p} 21$ was associated with greater CAD burden as marker of more severe atherosclerosis but not with prevalent myocardial infarction (21).

In this study, we aimed to identify genetic loci more specifically associated with the severity and distribution of CAD by using a two-stage approach including a discovery stage and a subsequent replication stage. While none of the SNPs reached genome-wide significance in the discovery stage, several SNPs on chromosome 2 and chromosome 9 showed borderline significance. In the replication phase, the locus on chromosome 2 could not be verified; however, our results reconfirmed the known chromosome 9p21 locus as locus for CAD severity and distribution.

Strengths of this study include the availability of data from three large CAD studies, independent replication of results,

TABLE 2 | Effects of selected SNPs in the combined discovery and replication phase.

\begin{tabular}{|c|c|c|c|c|c|c|c|c|c|c|}
\hline SNP ID & chr & Pos & $\begin{array}{l}\text { Coded } \\
\text { allele }\end{array}$ & $\begin{array}{l}\text { Other } \\
\text { allele }\end{array}$ & Nearest gene & $\begin{array}{l}\text { Frequency } \\
\text { coded } \\
\text { allele [\%] }\end{array}$ & \multicolumn{2}{|c|}{ Discovery } & \multicolumn{2}{|c|}{ Replication } \\
\hline rs1485086 & 2 & 139722479 & C & $G$ & $\begin{array}{l}\text { Upstream of NXPH2 } \\
\text { (184.7kb) }\end{array}$ & 9.5 & $0.196(0.040)$ & $7.82 \times 10^{-7}$ & $-0.079(0.043)$ & $6.63 \times 10^{-2}$ \\
\hline rs16835318 & 2 & 194659522 & A & $G$ & - & 8.8 & $0.191(0.041)$ & $3.83 \times 10^{-6}$ & $0.003(0.046)$ & $9.56 \times 10^{-1}$ \\
\hline rs17752803 & 2 & 139810489 & T & C & $\begin{array}{l}\text { Upstream of NXPH2 } \\
(272.7 \mathrm{~kb})\end{array}$ & 10.0 & $-0.214(0.042)$ & $4.20 \times 10^{-7}$ & $-0.061(0.042)$ & $1.48 \times 10^{-1}$ \\
\hline rs1333049 & 9 & 22125503 & C & $G$ & $\begin{array}{l}\text { Downstream of CDKN2B- } \\
\text { AS1 (4.4kb) }\end{array}$ & 49.0 & $0.101(0.024)$ & $1.86 \times 10^{-5}$ & $0.062(0.025)$ & $1.27 \times 10^{-2}$ \\
\hline
\end{tabular}

For single SNP replication analysis, we assumed concordant direction of effect and p-value $<0.05$. 


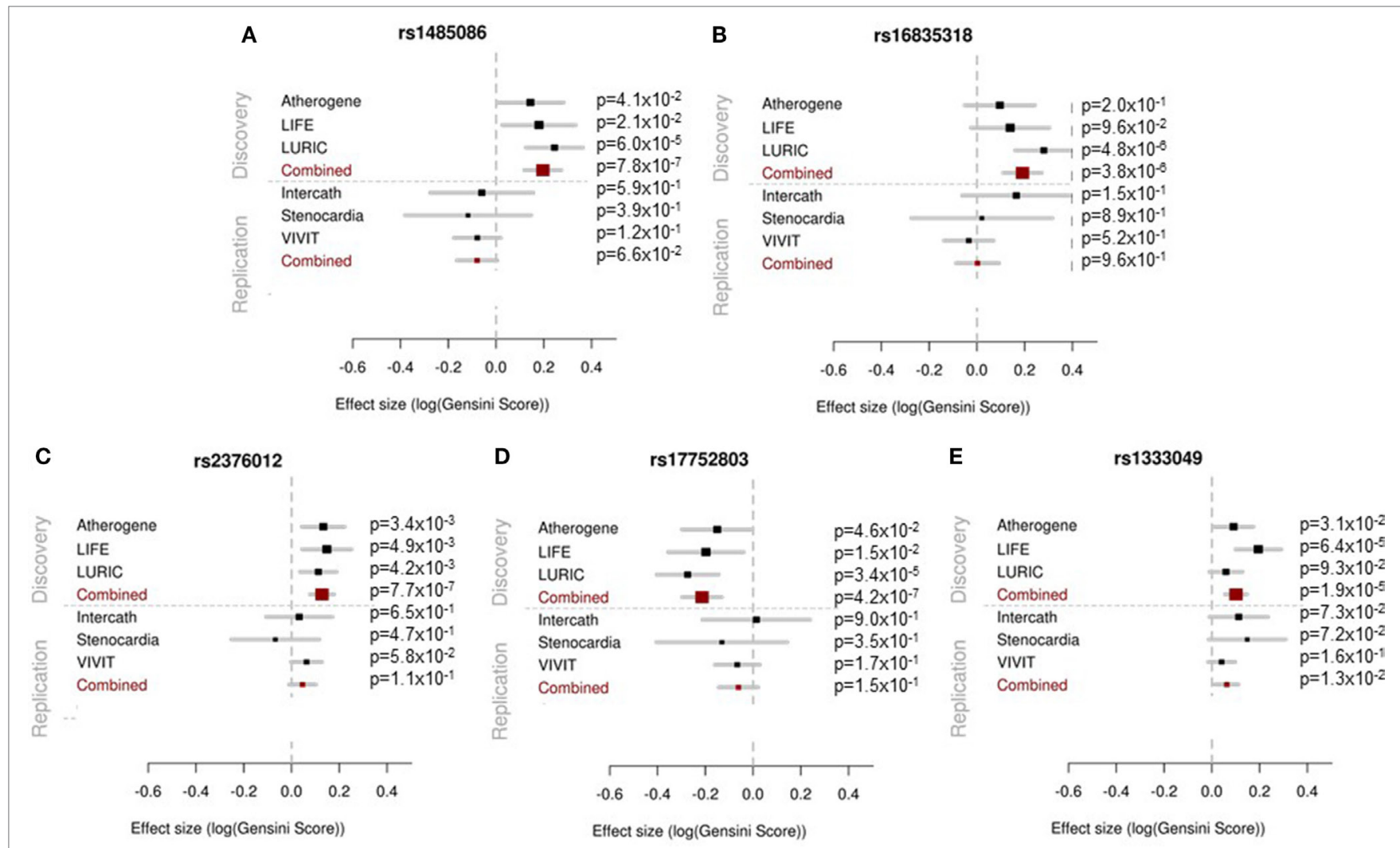

FIGURE 2 | Forest plots showing the results of the association analyses for each single study in the discovery (upper panel) and replication (lower panel) phase and for the meta-analysis are presented. The effect estimates and 95\% confidence intervals are provided for each study separately and in combined analysis.

and standardized definition and assessment of the extent of CAD through a validated semiquantitative score. Furthermore, similarity in quality control measures across cohorts, imputation strategies, and analytical methods account for homogeneity in analyses.

Some important limitations of our study should be noted. Compared to other GWAS samples, our combined samples were only of medium size because of the rigorous assessment of CAD that is not widely available in current GWAS studies. However, based on our statistical power, we should have been able to find medium to large effects with possible clinical relevance. Examination of larger cohorts with CAD defined by the Gensini Score may detect more genome-wide significant associations. In addition, we cannot generalize our findings to individuals of non-European ancestry. Assessment of CAD severity by semiquantitative angiographic scoring relies on angiographically detectable lesions. Disease burden that is limited to the vascular wall without significant intraluminal progression may be missed by this evaluation.

In summary, in-depth phenotyping of CAD using the angiographically determined Gensini score confirmed the chromosome 9p21 locus as risk locus of CAD severity. No additional locus of CAD severity was identified in this study. Genetic correlates of the CAD scoring systems need to be investigated in larger cohorts.

\section{ETHICS STATEMENT}

Each study was carried out in accordance with the recommendations of the local ethics committee, and all participants gave written informed consent in accordance with the Declaration of Helsinki. The protocols were approved by the respective local ethics and data safety committee. The cohorts are described in detail in the Supplementary Material.

\section{AUTHOR CONTRIBUTIONS}

TZ and MoS: study design, data acquisition, writing of manuscript, and critical revision of manuscript. CM: data analyses and meta analyses, and critical revision of manuscript. MS, FO, and MK: data analyses and critical revision of manuscript. AS, HD, AM, WM, CS, FB, CW, TK, CHS, KS, JT, DT: data acquisition and revision of manuscript. SB: study design, writing of manuscript and critical revision of manuscript. RS: study design, data acquisition, writing of manuscript, and critical revision of manuscript.

\section{FUNDING}

AtheroGene: The AtheroGene study was supported by a grant of the "Stiftung Rheinland-Pfalz für Innovation," Ministry 
for Science and Education (AZ 15202-386261/545), Mainz. This work was further supported by research grants from the Brandenburg Ministry of Economics, Germany, and the European Regional Development Fund (EFRE/ERDF). LURIC: The LURIC Study is supported by the European Union 7th Framework Program (AtheroRemo, grant agreement 201668, and RiskyCAD, grant agreement 305739), INTERREG-IVOberrhein-Program (project A28, Genetic Mechanisms of Cardiovascular Diseases), European Regional Development Fund (ERDF), Wissenschaftsoffensive TMO. LIFE Heart: we thank the patients of LIFE-Heart very much for their time and blood samples. LIFE Heart is funded by the Leipzig Research Center for Civilization Diseases (LIFE). LIFE is an organizational unit affiliated to the Medical Faculty of the University of Leipzig. LIFE is funded by means of the European Union, by the European Regional Development Fund (ERDF) and by funds of the Free

\section{REFERENCES}

1. McPherson R, Tybjaerg-Hansen A. Genetics of coronary artery disease. Circ Res (2016) 118:564-78. doi:10.1161/CIRCRESAHA.115.306566

2. Kessler T, Erdmann J, Schunkert H. Genetics of coronary artery disease and myocardial infarction - 2013. Curr Cardiol Rep (2013) 15:368. doi:10.1007/ s11886-013-0368-0

3. Nikpay M, Goel A, Won HH, Hall LM, Willenborg C, Kanoni S, et al. A comprehensive 1,000 Genomes-based genome-wide association meta-analysis of coronary artery disease. Nat Genet (2015) 47:1121-30. doi:10.1038/ng.3396

4. Wilson PW, D'agostino RB, Levy D, Belanger AM, Silbershatz H, Kannel WB. Prediction of coronary heart disease using risk factor categories. Circulation (1998) 97:1837-47. doi:10.1161/01.CIR.97.18.1837

5. Yeboah J, Mcclelland RL, Polonsky TS, Burke GL, Sibley CT, O’leary D, et al. Comparison of novel risk markers for improvement in cardiovascular risk assessment in intermediate-risk individuals. JAMA (2012) 308:788-95. doi:10.1001/jama.2012.9624

6. Montalescot G, Sechtem U, Achenbach S, Andreotti F, Arden C, Budaj A, et al. 2013 ESC guidelines on the management of stable coronary artery disease: the Task Force on the management of stable coronary artery disease of the European Society of Cardiology. Eur Heart J (2013) 34:2949-3003. doi:10.1093/eurheartj/eht296

7. Gensini GG. A more meaningful scoring system for determining the severity of coronary heart disease. Am J Cardiol (1983) 51:606. doi:10.1016/ S0002-9149(83)80105-2

8. Blankenberg S, Rupprecht HJ, Bickel C, Torzewski M, Hafner G, Tiret L, et al. Glutathione peroxidase 1 activity and cardiovascular events in patients with coronary artery disease. N Engl J Med (2003) 349:1605-13. doi:10.1056/ NEJMoa030535

9. Beutner F, Teupser D, Gielen S, Holdt LM, Scholz M, Boudriot E, et al. Rationale and design of the Leipzig (LIFE) Heart Study: phenotyping and cardiovascular characteristics of patients with coronary artery disease. PLoS One (2011) 6:e29070. doi:10.1371/journal.pone.0029070

10. Winkelmann BR, Marz W, Boehm BO, Zotz R, Hager J, Hellstern P, et al. Rationale and design of the LURIC study - a resource for functional genomics, pharmacogenomics and long-term prognosis of cardiovascular disease. Pharmacogenomics (2001) 2:S1-73. doi:10.1517/14622416.2.1.S1

11. Sinning C, Lillpopp L, Appelbaum S, Ojeda F, Zeller T, Schnabel R, et al. Angiographic score assessment improves cardiovascular risk prediction: the clinical value of SYNTAX and Gensini application. Clin Res Cardiol (2013) 102:495-503. doi:10.1007/s00392-013-0555-4

12. Diemert P, Schunkert H. Genomic reflections of coronary artery disease. Circ Cardiovasc Genet (2011) 4:7-8. doi:10.1161/CIRCGENETICS.110.959163

13. Fischer M, Broeckel U, Holmer S, Baessler A, Hengstenberg C, Mayer B, et al. Distinct heritable patterns of angiographic coronary artery disease in families with myocardial infarction. Circulation (2005) 111:855-62. doi:10.1161/01. CIR.0000155611.41961.BB
State of Saxony within the framework of the excellence initiative. VIVIT: The VIVIT study was supported by grants from the "Land Vorarlberg." stenoCardia: The stenoCardia study was supported by the "Schwerpunkt Vaskuläre Prävention" of the Johannes Gutenberg-University of Mainz. TZ is supported by the German Center for Cardiovascular Research (DZHK, 81Z1710101). RS is supported by the European Research Council under the European Union's Horizon 2020 research and innovation program (grant agreement no. 648131) and the German Ministry of Research and Education (BMBF 01ZX1408A).

\section{SUPPLEMENTARY MATERIAL}

The Supplementary Material for this article can be found online at http://journal.frontiersin.org/article/10.3389/fcvm.2017.00057/ full\#supplementary-material.

14. Dandona S, Stewart AF, Chen L, Williams K, So D, O'brien E, et al. Gene dosage of the common variant $9 \mathrm{p} 21$ predicts severity of coronary artery disease. J Am Coll Cardiol (2010) 56:479-86. doi:10.1016/j.jacc.2009.10.092

15. Patel RS, Su S, Neeland IJ, Ahuja A, Veledar E, Zhao J, et al. The chromosome 9 p21 risk locus is associated with angiographic severity and progression of coronary artery disease. Eur Heart J (2010) 31:3017-23. doi:10.1093/ eurheartj/ehq272

16. Rivera NV, Carreras-Torres R, Roncarati R, Viviani-Anselmi C, De Micco F, Mezzelani A, et al. Assessment of the 9p21.3 locus in severity of coronary artery disease in the presence and absence of type 2 diabetes. BMC Med Genet (2013) 14:11. doi:10.1186/1471-2350-14-11

17. Cakmak HA, Bayoglu B, Durmaz E, Can G, Karadag B, Cengiz M, et al. Evaluation of association between common genetic variants on chromosome 9p21 and coronary artery disease in Turkish population. Anatol J Cardiol (2015) 15:196-203. doi:10.5152/akd.2014.5285

18. Jing J, Su L, Zeng Y, Tang X, Wei J, Wang L, et al. Variants in 9p21 predicts severity of coronary artery disease in a Chinese han population. Ann Hum Genet (2016) 80:274-81. doi:10.1111/ahg.12163

19. Anderson JL, Horne BD, Kolek MJ, Muhlestein JB, Mower CP, Park JJ, et al. Genetic variation at the 9p21 locus predicts angiographic coronary artery disease prevalence but not extent and has clinical utility. Am Heart J (2008) 156(1155-1162):e1152. doi:10.1016/j.ahj.2008.07.006

20. Chen SN, Ballantyne CM, Gotto AM Jr, Marian AJ. The 9p21 susceptibility locus for coronary artery disease and the severity of coronary atherosclerosis. BMC Cardiovasc Disord (2009) 9:3. doi:10.1186/1471-2261-9-3

21. Chan K, Patel RS, Newcombe P, Nelson CP, Qasim A, Epstein SE, et al. Association between the chromosome 9p21 locus and angiographic coronary artery disease burden: a collaborative meta-analysis. J Am Coll Cardiol (2013) 61:957-70. doi:10.1016/j.jacc.2012.10.051

Conflict of Interest Statement: SB has received research funding from Abbott, Abbott Diagnostics, Bayer, Boehringer Ingelheim, SIEMENS, Singulex, and Thermo Fisher; honoraria for lectures from Abbott, Abbott Diagnostics, Astra Zeneca, Bayer, Boehringer Ingelheim, Medtronic, Pfizer, Roche, SIEMENS Diagnostics, SIEMENS, Thermo Fisher and as member of Advisory Boards and for consulting for Boehringer Ingelheim, Bayer, Novartis, Roche, and Thermo Fisher. The handling Editor declared a shared affiliation, though no other collaboration, with the authors MK and WM.

Copyright ( 2017 Zeller, Seiffert, Müller, Scholz, Schäffer, Ojeda, Drexel, Mündlein, Kleber, März, Sinning, Brunner, Waldeyer, Keller, Saely, Sydow, Thiery, Teupser, Blankenberg and Schnabel. This is an open-access article distributed under the terms of the Creative Commons Attribution License (CC BY). The use, distribution or reproduction in other forums is permitted, provided the original author(s) or licensor are credited and that the original publication in this journal is cited, in accordance with accepted academic practice. No use, distribution or reproduction is permitted which does not comply with these terms. 